\title{
Efficacy of the Endoscopic Ultrasound-first Approach in Patients with Suspected Common Bile Duct Stone to Avoid Unnecessary Endoscopic Retrograde Cholangiopancreatography
}

\author{
Akinori Maruta ${ }^{1}$, Takuji Iwashita ${ }^{1}$, Shinya Uemura ${ }^{1}$, Kensaku Yoshida ${ }^{1}$, \\ Ichiro Yasuda $^{2}$ and Masahito Shimizu ${ }^{1}$
}

\begin{abstract}
:
Objective Endoscopic ultrasound (EUS) is a safe and accurate examination for evaluating the presence of common bile duct stones (CBDSs). The EUS-first approach, where EUS is performed before endoscopic retrograde cholangiopancreatography (ERCP) for patients suspected of having CBDSs, may help reduce the risk of ERCP-related adverse events and save manpower by avoiding unnecessary ERCP. To evaluate the efficacy of the EUS-first approach in patients with suspected CBDSs.

Methods Between April 2012 and March 2016, 104 patients who underwent the EUS-first approach for suspected CBDSs were retrospectively evaluated. The relevant outcomes were the short- and long-term adverse event rates and the ERCP avoidance rate.

Results EUS findings were positive for CBDSs in 52 patients, showed sludge formation or possible CBDSs in 4 patients, and were negative for CBDSs in 42 patients (but positive for other diseases in 6). Sixty-two patients $(62 / 104,59.6 \%)$ underwent ERCP, and proper treatments were successfully performed in all but 1 who underwent only cholangiography. In the remaining 42 patients $(42 / 104,40.4 \%)$, ERCP was omitted based on the EUS findings. Early adverse events were recognized in $0 \%$ of the EUS-only group and 8 patients $(12.9 \%)$ in the EUS+ERCP group ( $\mathrm{p}=0.02$ ). Regarding late adverse events, recurrent CBDSs occurred in 1 patient $(2.3 \%)$ in the EUS-only group and $2(3.2 \%)$ in the EUS+ERCP group $(\mathrm{p}=1.0)$.

Conclusion The EUS-first approach in patients with suspected CBDSs was useful for reducing early adverse events associated with ERCP without increasing the late adverse event rate, as EUS enabled the avoidance of unnecessary ERCP.
\end{abstract}

Key words: endoscopic ultrasound, common bile duct stone, stone management, adverse event

(Intern Med 58: 1673-1679, 2019)

(DOI: 10.2169/internalmedicine.2047-18)

\section{Introduction}

Common bile duct stones (CBDSs) are frequently encountered in daily clinical practice. Since CBDSs can eventually cause serious adverse events, such as acute cholangitis or acute biliary pancreatitis, the basic management is the removal of the stone from the CBD, even if patients are asymptomatic when the stone is detected. Endoscopic retro- grade cholangiopancreatography (ERCP) has been the goldstandard diagnostic procedure for CBDSs, offering an advantage over other diagnostic procedures in that it allows for subsequent therapeutic intervention for CBDSs upon a positive diagnosis via cholangiography. However, ERCP does not have a high sensitivity for the detection of small CBDSs (1), and the procedure itself is often associated with serious adverse events, such as post-ERCP pancreatitis (2). Therefore, ideally, the presence of CBDSs should be con-

${ }^{1}$ First Department of Internal Medicine, Gifu University Hospital, Japan and ${ }^{2}$ Department of Gastroenterology, Teikyo University Mizonokuchi Hospital, Japan

Received: August 28, 2018; Accepted: December 9, 2018; Advance Publication by J-STAGE: February 25, 2019

Correspondence to Dr. Takuji Iwashita, takuji@w7.dion.ne.jp 


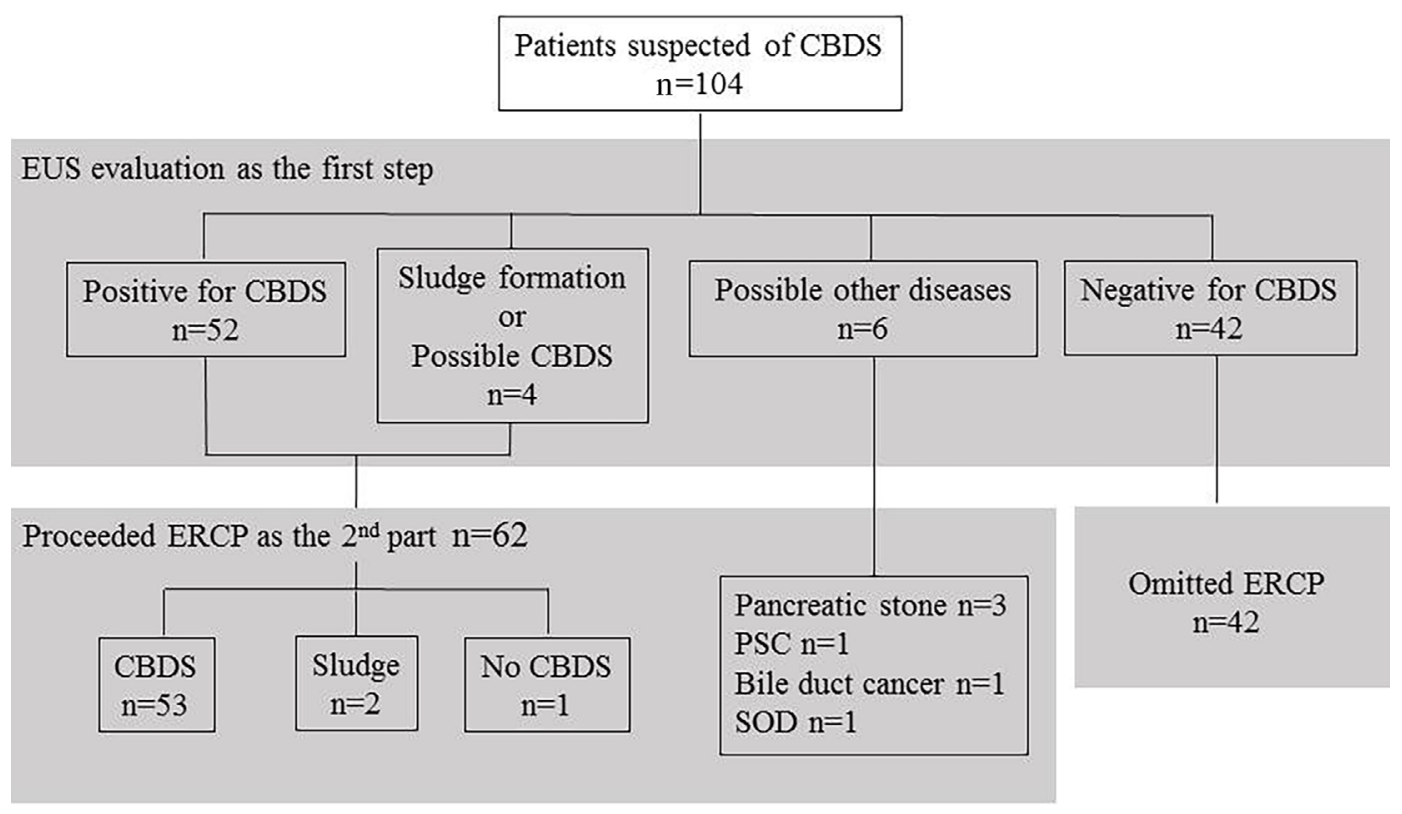

Figure 1. The step-wise diagnosis of patients with suspected common bile duct stones. CBDS: common bile duct stone, EUS: endoscopic ultrasound, ERCP: endoscopic retrograde cholangiopancreatography, PSC: primary sclerosing cholangitis, SOD: sphincter of Oddi dysfunction

firmed by a less-invasive examinations before initiating ERCP.

Endoscopic ultrasound (EUS) is a much-less-invasive diagnostic method reported to have very high diagnostic performance for CBDSs (3). The EUS-first approach, whereby EUS is performed before ERCP to evaluate the pancreatobiliary system, has been adopted in our institution for assessing patients suspected of having CBDSs. The EUS-first approach may aid in deciding whether or not a patient should undergo ERCP. For example, if CBDSs are noted on EUS, the patient is indicated for ERCP with stone removal, with ample information regarding the number and size of the stones; if CBDSs are not noted on EUS, the indication for ERCP should be reconsidered. Such a stepwise decisionmaking strategy may help reduce the risk for ERCP-related adverse events as well as save manpower by avoiding unnecessarily performing procedures.

We conducted the present study to evaluate the effectiveness of the EUS-first approach in patients with suspected CBDSs.

\section{Materials and Methods}

\section{Patients}

This was a single-center, retrospective, cohort study undertaken at Gifu University Hospital. The analysis included data regarding patients with suspected CBDS who underwent EUS and subsequent ERCP investigations between April 2012 and March 2016 (Fig. 1). Patients with a history of surgery of the upper intestine were excluded. A suspicion of CBDSs was defined in terms of the following findings: abnormal results of liver function tests, with symptoms such as abdominal pain; a possible CBDS diagnosis based on imaging findings, such as hyperechoic spots on abdominal ultrasound (AUS), high-signal-intensity areas on computed tomography (CT), defect on magnetic resonance cholangiopancreatography (MRCP), a low-signal-intensity area on both T1- and T2-weighted magnetic resonance imaging (MRI) or a high-signal-intensity area on T1-weighted MRI and a low-signal-intensity area T2-weighted MRI; a suspicion of biliary pancreatitis due to acute pancreatitis associated with abnormal liver function tests or possible CBDS on imaging studies (Fig. 2A).

Written informed consent to perform all procedures was obtained from all patients. This study was approved by the Institutional Review Board of our hospital.

\section{EUS-first approach}

All endoscopic procedures were performed by endoscopists with ample experience in both EUS- and ERCP-related procedures and took place in a fluoroscopy room equipped with EUS capabilities. A radial or convex ultrasonic gastrovideoscope (GF-UE260-AL5, GF-UC240P-AL5, or GFUCT260; Olympus, Tokyo, Japan) was used for EUS examinations, and a duodenovideoscope (TJF-260V; Olympus) was used for ERCP-related procedures.

In the EUS-first approach, the biliary duct from the duodenal ampulla to the common hepatic duct as well as the gallbladder and pancreas were evaluated via the stomach and the first and second parts of the duodenum using push and pull scope positions during an EUS examination. Patients with EUS findings consisting of hyperechoic structures with acoustic shadow inside the biliary duct were defined as positive for CBDSs. The number and diameter of the identified stones as well as the size of the CBD were 

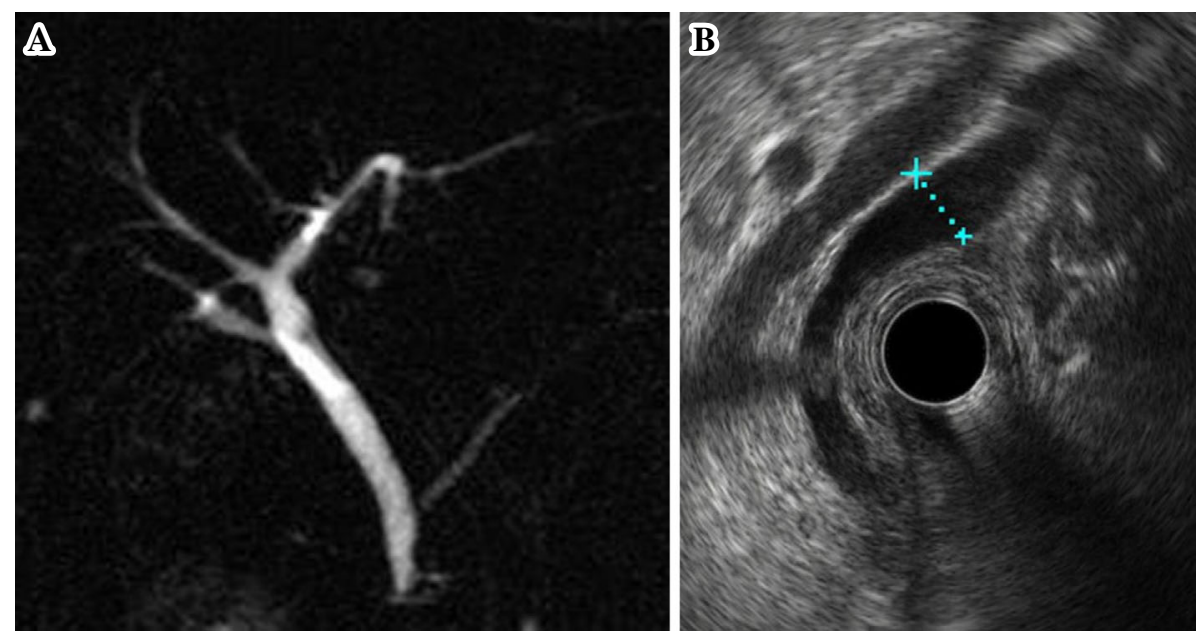

Figure 2. (A) Magnetic resonance cholangiopancreatography did not show any obvious biliary stones, although one patient had abnormal findings of liver function tests with abdominal pain. (B) However, EUS clearly showed a hyperechoic structure with acoustic shadow, measuring $4 \mathrm{~mm}$ in diameter. The stone was successfully managed by subsequent ERCP.

evaluated. Patients with EUS findings indicative of CBDSs underwent subsequent ERCP, whereas ERCP was omitted in those with CBDS-negative EUS findings (Fig. 2B). Subsequent ERCP consisted of initial cholangiography followed by the evaluation of the size and number of stones as well as the size of the CBD. A guidewire was then inserted into the biliary system in order to enable endoscopic sphincterotomy or papillary large balloon dilation, depending on the cholangiography findings. The stones were removed using a retrieval balloon or extraction basket. All patients underwent ERCP as inpatients and blood examinations at 2 hours after the procedure as well as on the first postoperative day in order to monitor potential adverse events.

\section{Data analyses}

The primary outcomes measured in the study were the incidence of early ( $\leq 14$ days) and late ( $>14$ days) adverse events after the EUS-based diagnosis. The secondary outcome was the rate of omitted ERCP in the EUS-first approach. Early adverse events after EUS were defined based on the lexicon for endoscopic adverse events described by the American Society Gastrointestinal Endoscopy (ASGE) (4). Late adverse events were defined as any events associated with the biliary system.

The study population was stratified into two groups based on whether or not ERCP was performed following the EUSbased diagnosis (EUS-only vs. EUS+ERCP). The EUS-only and EUS+ERCP groups were compared in terms of the incidence of adverse events. Continuous variables are presented in terms of the median and interquartile range (IQR). The rate of omitted ERCP was calculated along with its 95\% confidence interval $(95 \% \mathrm{CI})$. Comparisons were performed using Fisher's exact test for categorical variables and the Mann-Whitney U test for continuous variables. A 2-sided $\mathrm{p}$ value of $<0.05$ was considered to indicate statistical significance. All statistical analyses were performed using the JMP software program, version 10 (SAS Institute, Cary, USA).

\section{Results}

\section{Basic characteristics}

Our retrospective analysis of the hospital's database identified 250 patients with CBDSs and 104 patients (55 men, 49 women) suspected of having CBDSs who were treated via an EUS-first approach [median age of 72 years (IQR, 64-79 years)]. All patients underwent $\geq 1$ imaging investigation, as follows: AUS in 18 patients, CT in 96 patients, and MRCP in 22 patients. CBDSs were suspected based on imaging findings in 59 patients, abnormal liver function tests with symptoms in 29 patients, and suspicion of biliary pancreatitis in 16 patients. No patients underwent direct ERCP for suspected CBDS in this study period (Table 1).

\section{Results of EUS}

The EUS evaluation enabled the successful assessment of the CBD in all patients; a radial EUS scope was used in 91 patients, while a convex EUS scope was used in 13 patients. EUS showed CBD with a median diameter of $5 \mathrm{~mm}$ (IQR, 4-8 mm). A total of 52 and 42 patients were diagnosed as CBDS-positive and CBDS-negative, respectively; sludge formation or possible CBDSs was noted in 4 patients, and another 6 patients were assessed as positive for other diseases. EUS findings in the gallbladder were positive for stones in 64 patients and negative for stones in 26 patients; postcholecystectomy status was noted in 10 patients; in 4 patients, the gallbladder could not be successfully evaluated. EUS required a median of 8 minutes (IQR, 5-11) for the evaluation of the pancreatobiliary system. Following the EUS-based evaluation, a total of 62 patients $(62 / 104,59.6 \%)$ underwent ERCP for the management of CBDSs and further investigation of sludge, possible CBDS, or other diseases 
(suspected stone in the main pancreatic duct in 3 patients; thickened wall of the biliary duct in 2 patients; sphincter of Oddi dysfunction suspected based on recurrent episodes of abdominal pain associated with elevated levels of liver enzymes in 1 patient). The diagnostic yield of EUS for CBDSs in this study was $98.2 \%(55 / 56)$. In the remaining 42 patients (42/104, 40.4\%; 95\% CI, 31.5-50.0\%), ERCP was omitted based on the EUS findings (Table 2). Of the 42 patients with negative CBDSs findings on EUS, 5 had a history of cholecystectomy at the time of the examination. Cholecystolithiasis was recognized in 22 patients, 7 of whom underwent subsequent cholecystectomy. During the median follow-up period of 5 months (IQR: 1-22), CBDSs were diagnosed in 1 (2.4\%) of the 42 patients negative for CBDSs on EUS. In that 1 patient, CBDSs were diagnosed 25 months after EUS and were considered to have newly migrated from the gallbladder based on CT findings. The patient eventually underwent cholecystectomy after endoscopic management of CBDSs.

Table 1. Baseline Characteristics of Patients with Suspected Common Bile Duct Stone.

\begin{tabular}{lc}
\hline Male / Female, $\mathrm{n}$ & $55 / 49$ \\
Age, year-old, median (IQR) & 72 yo (64-79) \\
Diagnostic imaging & \\
-AUS, $\quad$ suspicious / negative & $6 / 12$ \\
-CT, suspicious / negative & $55 / 41$ \\
-MRI / MRCP, suspicious / negative & $13 / 9$ \\
Reasons for suspected CBDS, n (\%) & \\
-possible CBDS on imaging findings & $58(55.8 \%)$ \\
-abnormal LFTs with symptoms & $30(28.8 \%)$ \\
-possible biliary pancreatitis & $16(15.4 \%)$ \\
\hline IQR: interquartile range, CBDS: common bile duct stone, LFT: \\
liver function test
\end{tabular}

\section{Results of ERCP}

Biliary duct cannulation was successful in all 62 patients who underwent ERCP, and cholangiography showed a CBD with a median diameter of $8.2 \mathrm{~mm}$ (IQR, 6-11 mm). CBDSpositive findings were noted in 53 patients, with a median stone diameter of $5 \mathrm{~mm}$ (IQR, 4-7 mm). Among the 4 patients with sludge formation or possible CBDSs on EUS, cholangiography confirmed CBDSs in 1 patient, indicated a possible defect in 2 patients, and detected no CBDS findings in 1 patient. All patients received further treatment of the confirmed biliary disease except for the one patient with suspected CBDSs on EUS but negative findings on ERCP. CBDSs or sludge were successfully removed using an extraction basket or retrieval balloon after sphincterotomy in 46 patients or endoscopic papillary large balloon dilation in 6 patients; in 3 patients, no additional interventions for the papilla were performed because of the patients' history of sphincterotomy. Regarding the six patients with non-CBDS diseases: pancreatic stones were endoscopically removed after pancreatic sphincterotomy in three; sphincterotomy followed by a biliary biopsy confirmed the diagnosis of bile duct cancer in one; cholangiography and a negative biliary biopsy after sphincterotomy were compatible with primary sclerosing cholangitis in one; and sphincterotomy alone was performed in one patient suspected of having sphincter of Oddi dysfunction. ERCP, including management of biliary disease, required a median of 20 minutes (IQR, 15-26 minutes) (Table 3).

\section{Adverse events}

No early adverse events were noted in the EUS-only group, whereas early adverse events were recognized in 8 patients $(12.9 \%)$ of the EUS+ERCP group ( $\mathrm{p}=0.02)$ and consisted of mild abdominal pain (4 patients) and post-

Table 2. Results of the Endoscopic Ultrasound Investigation in Patients with Suspected Common Bile Duct Stone.

\begin{tabular}{lc}
\hline Successful EUS evaluation of the CBD, n (\%) & $104(100 \%)$ \\
Type of scope in EUS, n (\%) & \\
-Radial type & $91(87.5 \%)$ \\
-Convex type & $13(12.5 \%)$ \\
Diameter of CBDS, mm, median (IQR) & $5 \mathrm{~mm}(4-8)$ \\
Findings in the CBD, n (\%) & \\
-positive for CBDS & $52(50.0 \%)$ \\
-sludge formation or possible CBDS & $4(3.8 \%)$ \\
-positive for other diseases & $6(5.8 \%)$ \\
-negative for CBDS & $42(40.4 \%)$ \\
Findings in the gall bladder, n (\%) & \\
-positive for GBS & $64(61.5 \%)$ \\
-negative for GBS & $26(25.0 \%)$ \\
-status post-cholecystectomy & $10(9.6 \%)$ \\
-poor evaluation & $4(3.8 \%)$ \\
Time required for EUS evaluation, min, median (IQR) & $8 \mathrm{~min}(5-11)$ \\
\hline
\end{tabular}

CBD: common bile duct, CBDS: common bile duct stone, EUS: endoscopic ultrasound, GBS: gallbladder stone 
ERCP pancreatitis (mild, 3 patients; moderate, 1 patient). All early adverse events were successfully managed conservatively. Late adverse events were recognized in 3 patients. Patients in the EUS-only group were followed-up for a median of 5 months (IQR, 1-22 months), and 1 patient (2.3\%) was diagnosed with CBDSs 2 months after the procedure. Patients in the EUS+ERCP group were followed for a median of 11 months (IQR, 1-22 months), and 2 patients (2/62, $3.2 \%$ ) developed recurrent CBDS at 9 and 26 months after ERCP (Table 4).

\section{Discussion}

In the diagnosis of CBDSs, imaging modalities such as AUS, CT, and MRCP have been employed as easy-toperform and noninvasive diagnostic methods; however, the reported diagnostic yields for CBDSs using such imaging

Table 3. Results of Endoscopic Retrograde Cholangiography.

\begin{tabular}{lc}
\hline Successful biliary cannulation, n (\%) & $62(100 \%)$ \\
Diameter of the CBD, mm median(IQR) & $8.2(6-11)$ \\
Findings of ERCP, n (\%) & $53(85.5)$ \\
-CBDS & $2(14.5)$ \\
-sludge formation & $3(4.8 \%)$ \\
-pancreatic stones & $1(1.6 \%)$ \\
-bile duct cancer & $1(1.6 \%)$ \\
-PSC & $1(1.6 \%)$ \\
-SOD & $1(1.6 \%)$ \\
-no abnormal findings & $46(74.2 \%)$ \\
Therapeutic methods, n (\%) & $2(3.2 \%)$ \\
-EST with CBDS/sludge removal & $1(1.6 \%)$ \\
-EST with biopsy & $6(9.7 \%)$ \\
-EST only & $3(4.8 \%)$ \\
-EPLBD with stone removal & $1(1.6 \%)$ \\
-stone removal s/p EST/EPLBD & $3(4.8 \%)$ \\
-cholangiography only & 20 min $(15-26)$ \\
-EPST with pancreatic stone removal & \\
Time required for ERCP, min, median (IQR) & \\
\hline CBD: common bile duct, IQR: interquartile range, CBDS: common bile \\
duct stone, PSC: primary sclerosing cholangitis, SOD: sphincter of Oddi \\
papillary large balloon dilation, EPST: endoscopic pancreatic sphincter- \\
otomy, s/p status post
\end{tabular}

modalities are not very high. Regarding AUS, intestinal gas in the duodenum and obesity can impair the evaluation of the entire CBD, and the pooled diagnostic yield of 5 studies was reported to be $73 \%$ (5). Regarding CT, although intestinal gas and the patient's condition do not affect the imaging itself, the diagnostic yields for CBDSs were reported to range from $50-90 \%$ (6-8) because radio-transparent CBDSs cannot be visualized. The reported diagnostic yields of CBDS on MRCP were better than those of AUS or CT (85$100 \%$ ) (9-14), although MRCP had poor sensitivity for small CBDSs [diameter $<5 \mathrm{~mm}$ (15) or $<6 \mathrm{~mm}$ (16)].

In contrast, EUS can visualize the entire extrahepatic bile duct with very high resolution from the duodenum without any interference from intestinal gas or abdominal fat. The diagnostic yields of EUS for the detection of CBDSs are high, with a reported sensitivity of 0.94 and specificity of 0.95 according to a meta-analysis of studies including a total of 2,673 patients (17). In a prospective study comparing the diagnostic capability of imaging studies for CBDS, the sensitivities of EUS, MRCP, and drip infusion cholecystocholangiography (DIC)-CT have been $100 \%, 88 \%$, and $88 \%$, respectively. However, false-negative findings were detected with MRCP and DIC-CT in cases where the CBDSs were smaller than $5 \mathrm{~mm}$ in diameter (18). One drawback of EUS is that it is a relatively invasive procedure compared with other imaging modalities and requires patients be sedated for the duration of the procedure, although the incidence of adverse events related to this procedure is very low (19). Therefore, in patients suspected of having CBDSs, the EUSfirst approach represents a reasonable strategy as it is a much-less-invasive and more accurate diagnostic modality for CBDSs than other available approaches, and patients who ultimately require subsequent ERCP (for treatment or further investigation) will already have been sedated.

In the present study, the EUS-first approach was performed for 104 patients suspected of having CBDSs. A total of 64 patients underwent subsequent ERCP based on EUS findings of CBDSs or sludge with possible CBDSs $(n=56)$ and possible other diseases $(n=6)$. However, ERCP was omitted in 42 patients $(40.4 \%)$ in whom EUS did not show any abnormal findings. During ERCP, cholangiography followed by the successful management of CBDSs or sludge formation was performed in 55 patients, while cholangiogra-

Table 4. Adverse Events.

\begin{tabular}{lccc}
\hline & $\begin{array}{c}\text { EUS-only group } \\
(\mathrm{n}=42)\end{array}$ & $\begin{array}{c}\text { EUS+ERCP group } \\
(\mathrm{n}=62)\end{array}$ & p value \\
\hline Early adverse events, $\mathrm{n}(\%)$ & $0(0 \%)$ & $8(12.9 \%)$ & 0.02 \\
-mild pancreatitis & 0 & 3 & \\
-moderate pancreatitis & 0 & 1 & \\
-mild abdominal pain & 0 & 4 & \\
Late adverse events, $\mathrm{n}(\%)$ & $1(2.3 \%)$ & $2(3.2 \%)$ & 1.00 \\
-recurrent CBDS & 1 & 2 & \\
Follow-up period, months, median (IQR) & $5(1-22)$ & $11(1-22)$ & 0.19 \\
\hline
\end{tabular}

CBDS: common bile duct stone, IQR: interquartile range 
phy alone was performed in 1 patient in whom EUS findings were positive for CBDSs while cholangiography findings were negative. Therefore, the positive predictive value of EUS for detecting CBDSs in this study was 98.2\% (55/ 56). Therapeutic or diagnostic ERCP was performed in all six patients suspected of having other diseases on EUS. The early adverse event rate was significantly higher in the EUS+ERCP group than in the EUS-only group $(\mathrm{p}<0.02)$, although the late adverse event rate was similar between the groups $(\mathrm{p}=1.0)$. In our study, the EUS-first approach was able to reduce the rate of early adverse events, since ERCP was omitted in about $40 \%$ of patients suspected of having CBDSs.

There have been several prospective studies comparing "the EUS-first and the ERCP-second approach" and "the ERCP-first approach" in patients suspected of having CBDSs $(20,21)$. A study by Polkowski et al. (20) including 50 patients diagnosed via the EUS-first approach and 48 diagnosed via the ERCP-first approach compared negative outcomes related to either endoscopic procedures or a falsenegative diagnosis of CBDSs. In that study, $10 \%$ of patients in the EUS-first group (5/50) and $40 \%$ of those in the ERCP-first group (19/48) experienced a negative outcome (p $<0.001$ ). Karakan et al. (21) also prospectively compared the EUS-first $(n=60)$ and ERCP-first $(n=60)$ approaches in patients suspected of having CBDS and reported a higher adverse event-free survival rate in the EUS-first group ( $\mathrm{p}=$ 0.049). The results of these two prospective studies suggest that the EUS-fist approach can reduce the incidence of early adverse events (mainly related to ERCP), as unnecessary ERCP can be avoided based on EUS findings. Furthermore, the EUS-first approach cannot increase the risk of late adverse events because EUS can accurately evaluate the CBD. These conclusions are in agreement with the results of our study, wherein the EUS-first approach was able to reduce the incidence of early adverse events without increasing the incidence of late adverse events.

According to the Japanese guidelines for the management of acute pancreatitis [Japanese Guidelines 2015 (22)], early ERCP with or without sphincterotomy is recommended for acute pancreatitis, which is diagnosed or suspected as gallstone-induced pancreatitis in cases of 1) complications associated with cholangitis or 2) a suspected prolonged passage disorder, such as in the development and/or deterioration of jaundice. In "the EUS-first approach", ERCP is performed for patients in whom CBDSs or bile duct obstruction was suspected. If pancreatitis does not seem to be related to CBDSs or bile duct obstruction, conservative treatment is prioritized, considering the risk of ERCP inducing further deterioration of pancreatitis. The present study enrolled 16 patients suspected of having biliary pancreatitis. ERCP was performed in nine of these patients because EUS showed CBDSs or biliary sludge in six patients and pancreatic stones in three. The further deterioration of pancreatitis was not recognized in any patients who underwent subsequent ERCP. In the remaining 7 patients, ERCP was omitted based on negative EUS findings, and acute pancreatitis was successfully managed with conservative treatment.

Another potential advantage of the EUS-first approach in patients suspected of having CBDSs is that the pancreatobiliary system can be accurately evaluated immediately prior to ERCP. In our study, 6 of 104 patients $(5.8 \%)$ had nonCBDS diagnoses (pancreatic stone in 3 patients, PSC in 1 patient, bile duct cancer in 1 patient, and sphincter of Oddi dysfunction in 1 patient). Some of those diseases might have been overlooked if the patients had undergone only ERCP for the detection of CBDSs; in particular, pancreatography is typically avoided in order to minimize the risk of postERCP pancreatitis, which may lead to overlooking pancreatic stones (2). Another point is that small CBDSs might be overlooked in the ERCP-first approach, since the reported sensitivity of ERCP for small CBDSs is lower than that of EUS (ERCP vs. EUS: 75\% vs. 91\%) (21). The therapeutic stage of ERCP (sphincterotomy or papillary balloon dilation) carries higher risks of adverse events than the diagnostic ERCP, so unnecessary intervention should be avoided if cholangiography fails to reveal small CBDSs. The EUS-first approach might be useful for reducing the possibility of oversight of small CBDSs and subsequent mismanagement.

However, there are several possible disadvantages of the EUS-first approach. First, while EUS can provide a highresolution assessment of the CBD from the duodenum, without the interference of intestinal gas or visceral fat, it cannot fully evaluate the hepatic hilum or intrahepatic bile duct and thus may lead to oversight of CBDSs that have migrated upstream into the biliary duct, beyond the hepatic hilum. In contrast, ERCP can evaluate the entire biliary system, and the success rate of CBDS therapy using ERCP exceeds $90 \%$, regardless of the presence of cholangitis or papillitis. In fact, one patient in whom the ERCP was omitted developed cholangitis due to recurrent CBDS; however, we believe that this condition might have been caused by a gallbladder stone, since cholecystectomy for gallstones was not performed in this patient, in accordance with their wishes. Second, the EUS-first approach may involve additional invasive procedures for treating the patient and may require additional manpower and time to perform EUS if the cohort has a high probability of CBDSs. However, it is difficult to accurately predict the provability for CBDS. According to the ASGE guideline regarding the role of endoscopy in the evaluation of patients suspected of having CBDSs (19), even in a cohort at high risk for having CBDSs, the estimated provability of CBDSs was not very high (reportedly $>50 \%$ ). Indeed, in the present study, the CBDS detection rates by EUS were $37.5 \%(6 / 16)$ in the low-risk cohort, $54.7 \%$ (41/ $75)$ in the intermediate-risk cohort, and $69.2 \%(9 / 13)$ in the high-risk cohort, according to the risk stratification by the ASGE guideline. Considering the high adverse event rate of ERCP, high diagnostic yield of EUS for CBDSs, and difficulty predicting the provability for CBDSs, we still feel it is reasonable to perform EUS before ERCP in patients suspected of having CBDSs, since EUS only took a median of 
8 minutes in our study for the evaluation of the pancreatobiliary system and led to no adverse events.

Several limitations associated with the present study warrant mention. First, this was a single-center study with a retrospective design, so our data may be biased in terms of the patient selection and the characteristics of the EUS and ERCP procedures. Furthermore, there was no specific protocol for the management of patients suspected of having CBDSs, and various imaging studies were performed, which made it difficult to stratify the patients in terms of their risk for CBDSs based on imaging findings.

Despite its limitations, our study brings clear evidence that the EUS-first approach helps avoid unnecessary ERCP and thereby reduces the incidence of early adverse events associated with ERCP in patients suspected of having CBDSs.

The authors state that they have no Conflict of Interest (COI).

\section{References}

1. Ney MV, Maluf-Filho F, Sakai P, Zilberstein B, Gama-Rodrigues J, Rosa H. Echo-endoscopy versus endoscopic retrograde cholangiography for the diagnosis of choledocholithiasis: the influence of the size of the stone and diameter of the common bile duct. Arq Gastroenterol 42: 239-243, 2005.

2. Freeman ML, Nelson DB, Sherman S, et al. Complications of endoscopic biliary sphincterotomy. N Engl J Med 335: 909-918, 1996.

3. Canto MI, Chak A, Stellato T, Sivak MV Jr. Endoscopic ultrasonography versus cholangiography for the diagnosis of choledocholithiasis. Gastrointest Endosc 47: 439-448, 1998.

4. Cotton PB, Eisen GM, Aabakken L, et al. A lexicon for endoscopic adverse events: report of an ASGE workshop. Gastrointest Endosc 71: 446-454, 2010.

5. Gurusamy KS, Giljaca V, Takwoingi Y, et al. Ultrasound versus liver function tests for diagnosis of common bile duct stones. Cochrane Database Syst Rev, 2015 (Epub ahead of print).

6. Mitchell SE, Clark RA. A comparison of computed tomography and sonography in choledocholithiasis. AJR Am J Roentgenol 142: 729-733, 1984

7. Jeffrey RB, Federle MP, Laing FC, Wall S, Rego J, Moss AA. Computed tomography of choledocholithiasis. AJR Am J Roentgenol 140: 1179-1183, 1983.

8. Baron RL. Common bile duct stones: reassessment of criteria for CT diagnosis. Radiology 162: 419-424, 1987.

9. Guibaud L, Bret PM, Reinhold C, Atri M, Barkun AN. Bile duct obstruction and choledocholithiasis: diagnosis with MR cholangiography. Radiology 197: 109-115, 1995.
10. Chan YL, Chan AC, Lam WW, et al. Choledocholithiasis: comparison of MR cholangiography and endoscopic retrograde cholangiography. Radiology 200: 85-89, 1996.

11. Pavone P, Laghi A, Catalano C, Broglia L, Fiocca F, Passariello R. Non-invasive evaluation of the biliary tree with magnetic resonance cholangiopancreatography: initial clinical experience. Ital J Gastroenterol 28: 63-69, 1996.

12. de Ledinghen V, Lecesne R, Raymond JM, et al. Diagnosis of choledocholithiasis: EUS or magnetic resonance cholangiography? A prospective controlled study. Gastrointest Endosc 49: 26-31, 1999.

13. Materne R, Van Beers BE, Gigot JF, et al. Extrahepatic biliary obstruction: magnetic resonance imaging compared with endoscopic ultrasonography. Endoscopy 32: 3-9, 2000.

14. Hochwald SN, Dobryansky MB, Rofsky NM, et al. Magnetic resonance cholangiopancreatography accurately predicts the presence or absence of choledocholithiasis. J Gastrointest Surg 2: 573-579, 1998.

15. Jendresen MB, Thorboll JE, Adamsen $S$, Nielsen H, Gronvall S, Hart-Hansen O. Preoperative routine magnetic resonance cholangiopancreatography before laparoscopic cholecystectomy: a prospective study. Eur J Surg 168: 690-694, 2002.

16. Zidi SH, Prat F, Le Guen $O$, et al. Use of magnetic resonance cholangiography in the diagnosis of choledocholithiasis: prospective comparison with a reference imaging method. Gut 44: 118$122,1999$.

17. Tse F, Liu L, Barkun AN, Armstrong D, Moayyedi P. EUS: a meta-analysis of test performance in suspected choledocholithiasis. Gastrointest Endosc 67: 235-244, 2008.

18. Kondo S, Isayama $\mathrm{H}$, Akahane $\mathrm{M}$, et al. Detection of common bile duct stones: comparison between endoscopic ultrasonography, magnetic resonance cholangiography, and helical-computedtomographic cholangiography. Eur J Radiol 54: 271-275, 2005.

19. Maple JT, Ben-Menachem T, Anderson MA, et al. The role of endoscopy in the evaluation of suspected choledocholithiasis. Gastrointest Endosc 71: 1-9, 2010.

20. Polkowski M, Regula J, Tilszer A, Butruk E. Endoscopic ultrasound versus endoscopic retrograde cholangiography for patients with intermediate probability of bile duct stones: a randomized trial comparing two management strategies. Endoscopy 39: 296303, 2007.

21. Karakan T, Cindoruk M, Alagozlu H, Ergun M, Dumlu S, Unal S. EUS versus endoscopic retrograde cholangiography for patients with intermediate probability of bile duct stones: a prospective randomized trial. Gastrointest Endosc 69: 244-252, 2009.

22. Yokoe M, Takada T, Mayumi T, et al. Japanese guidelines for the management of acute pancreatitis: Japanese Guidelines 2015. J Hepatobiliary Pancreat Sci 22: 405-432, 2015.

The Internal Medicine is an Open Access journal distributed under the Creative Commons Attribution-NonCommercial-NoDerivatives 4.0 International License. To view the details of this license, please visit (https://creativecommons.org/licenses/ by-nc-nd/4.0/).

(C) 2019 The Japanese Society of Internal Medicine Intern Med 58: 1673-1679, 2019 